\title{
EL INFLUJO DE CARTERA VENCIDA COMO MEDIDA DE RIESGO DE CREDITO: ANALISIS Y APLICACION AL CASO DE CHILE*
}

\author{
THE INFLOW INTO THE NON-PERFORMING LOANS RATIO \\ AS A MEASURE OF CREDIT RISK: ANALYSIS AND APPLICATION \\ IN THE CHILEAN CASE
}

\section{ANDRES SAGNERT.}

Banco Central de Chile

\begin{abstract}
This paper proposes the inflow into non-performing loans (INPL) ratio, defined as the change in the stock of non-performing loans adjusted by write-offs and standardized by loans, as the main measure to be used for modelling the credit risk of the Chilean banking system. In particular, the paper identifies certain statistical and conceptual advantages of this measure with respect to loan loss provisions (LLP), which support this idea. First, the INPL ratio by type of credit has a greater time span than LLP. Second, the forward-looking nature of LLP -one of its main advantages over the INPL ratio-is applicable only from 2004 onward due to various changes in Chilean reporting standards. Third, LLP is discretionary because provisioning is made on the basis of relative risk aversion of banks. Fourth, the INPL ratio produces smoother series than LLP for consumer and mortgage loans. In addition, the dynamic structure observed in both time series does not differ significantly. The econometric model estimated for the period January 1997 to June 2010 shows that the INPL ratio has statistically significant relations with macroeconomic aggregates such as the annual output growth, the short and long term interest rates, the annual inflation rate, the peso-dollar exchange rate, and non expected credit growth. Finally, the out-of-sample forecasts indicate differences between the actual and projected INPL ratios that are economically significant only in the case of mortgage credit. For the remaining portfolios, the evolution of this ratio during the period of
\end{abstract}

* Agradezco los valiosos comentarios y sugerencias de Rodrigo Alfaro, Herman Bennett, Rodrigo Cifuentes, Felipe Martínez, José Miguel Matus, Nancy Silva y un árbitro anónimo en la elaboración de este artículo. Cualquier error es de mi exclusiva responsabilidad. E-mail: asagner@bcentral.cl 
July 2008 to June 2010 does not differ significantly from that predicted by the econometric model.

Keywords: Credit risk, credit, loan accounting, general statistics, out-ofsample forecasts.

JEL Classification: C58, E51, G32.

\section{Resumen}

Este artículo propone el Influjo de Cartera Vencida (ICV), definido como la variación del stock de cartera vencida ajustada por castigos y normalizada por colocaciones, como principal medida a emplear para la modelación del riesgo de crédito del sistema bancario chileno. En particular, el artículo identifica ciertas ventajas estadísticas y conceptuales de esta medida, respecto del gasto en provisiones (GPR), que apoyan esta idea. En primer lugar, el ICV detallado por tipo de colocaciones posee una mayor cobertura temporal que el GPR. Segundo, la naturaleza forward-looking de GPR-una de sus principales ventajas sobre ICV-es aplicable sólo a partir del año 2004 debido a diversos cambios en la normativa chilena. Tercero, GPR es de carácter discrecional dado que la constitución de provisiones se realiza sobre la base de la aversión relativa al riesgo de los bancos evaluadores. Cuarto, el ICV genera series más suaves respecto del GPR para las carteras de consumo e hipotecaria. Por último, la estructura dinámica observada en ambas series no difiere de forma significativa. El modelo econométrico estimado para el período enero 1997 a junio 2010 revela que ICV posee relaciones estadísticamente significativas con agregados macroeconómicos como la variación anual del IMACEC, la tasa de interés de corto y largo plazo, la tasa de inflación anual, el tipo de cambio peso-dólar, y las sorpresas en el crecimiento de las colocaciones. Finalmente, las proyecciones fuera de muestra señalan diferencias económicamente significativas con el ICV efectivo sólo para el caso de las colocaciones hipotecarias. Para las carteras restantes, la evolución del índice durante el período julio 2008 a junio 2010 no difiere significativamente de aquella predicha por el modelo econométrico.

Palabras Clave: Riesgo de crédito, crédito, contabilidad del crédito, estadísticas generales, proyección fuera de muestra.

Clasificación JEL: C58, E51, G32. 


\section{INTRODUCCION}

Uno de los principales riesgos al cual las instituciones bancarias se ven expuestas corresponde al riesgo de crédito, el cual se define como la probabilidad de que un deudor bancario no pueda cumplir con sus obligaciones contractuales de intereses y/o capital (Matus, 2007). Si bien otros riesgos como el de tasas de interés, el de mercado y el operativo -entre muchos otros- han presentado una importancia creciente en el último tiempo debido a la mayor complejidad del negocio bancario nacional e internacional, existe abundante evidencia empírica que señala al riesgo de crédito como una de las principales causas de las crisis bancarias tanto de carácter sistémico como de carácter individual ${ }^{1}$.

La materialización de este tipo de riesgo suele generar pérdidas fiscales y reales elevadas, las que a su vez podrían afectar negativamente el desempeño del sector financiero y su estabilidad (Hoggarth, Reis y Saporta, 2001; Dell'Ariccia, Detragiache y Rajan, 2008). Es por esta razón que se torna relevante para los bancos centrales y/u organismos de supervisión bancaria disponer de medidas de riesgo de crédito adecuadas, que funcionen como indicadores de alerta temprana y que apoyen la toma de decisiones oportuna para minimizar la probabilidad de ocurrencia de crisis bancarias y sus potenciales costos. En este sentido, la literatura postula una serie de medidas de riesgo de crédito, siendo los indicadores por lejos más utilizados el gasto en provisiones, la cartera vencida y los castigos.

Este artículo propone el influjo de cartera vencida $(I C V)$, el cual se define como la variación del stock de cartera vencida ajustada por castigos y normalizada por colocaciones, como la principal medida de riesgo de crédito a emplear en el sistema bancario chileno. En particular, la preferencia del ICV por sobre el gasto en provisiones $(G P R)$-indicador de extensa utilización en Chile- se basa en tres ventajas asociadas al $I C V$. Primero, el influjo de cartera vencida detallado por tipo cartera posee una mayor cobertura temporal que GPR. Segundo, y si bien el gasto en provisiones es por definición un indicador de naturaleza forward-looking, dicha propiedad es válida en Chile sólo a partir del año 2004. Tercero, GPR es de carácter discrecional, en el sentido que la evaluación de riesgos bajo esta medida depende de la aversión relativa al riesgo de los bancos. En adición, el análisis de las propiedades estadísticas de ambas series revela que para la cartera de consumo e hipotecaria, ICV genera series más suaves que GPR mientras que, en términos globales, la estructura dinámica de ambas medidas no es estadísticamente distinta.

Los resultados asociados al modelo empírico que relaciona el influjo de cartera vencida con sus rezagos y un conjunto de agregados macroeconómicos durante el período enero 1997 a junio 2010 indican que: (i) para la cartera de consumo variables tales como la variación anual del IMACEC, la tasa de interés de corto plazo, la tasa anual de inflación y las sorpresas en el crecimiento de las colocaciones poseen efectos estadísticamente significativos sobre el ICV; (ii) el crecimiento económico y la variación trimestral del tipo de cambio peso-dólar son las únicas variables

1 Ver, por ejemplo, Goldstein y Turner (1996); Demirgüc-Kunt y Detragiache (1998); Hardy y Pazarbasioglu (1999); Ergungor y Thomson (2005) y Drehmann (2009). 
macroeconómicas que poseen efectos estadísticamente significativos sobre el ICV comercial, además de ser la medida que mayor grado de persistencia manifiesta ante variaciones inesperadas del riesgo de crédito comercial; y (iii) la tasa de interés de largo plazo y la variación anual del IMACEC poseen efectos económicamente significativos sobre el $I C V$ hipotecario, aunque dicha modelación es la que presenta los más bajos coeficientes de ajuste global ( $R^{2}$ ajustado en torno a $\left.33 \%\right)$. Por último, las proyecciones fuera de muestra obtenidas a partir de las especificaciones identificadas como favoritas indican diferencias estadísticamente significativas con el influjo de cartera vencida efectivo sólo para el caso de las colocaciones hipotecarias. Para las carteras restantes, la evolución del ICV durante el período julio 2008 a junio 2010 no difiere significativamente de aquella predicha por el modelo empírico.

El artículo se encuentra organizado de la siguiente forma. La Sección 2 presenta las principales medidas de riesgo de crédito disponibles en la literatura, y en base a sus definiciones y a la evolución de la normativa chilena, identifica las principales diferencias entre ellas. La Sección 3 define explícitamente el $I C V$, compara sus propiedades estadísticas con aquellas del gasto en provisiones y presenta el modelo econométrico que relaciona el influjo de cartera vencida -para cada tipo de colocaciones- con un conjunto de variables macroeconómicas. La Sección 4 discute los resultados obtenidos y revisa el poder predictivo de las especificaciones identificadas como favoritas. Finalmente, la Sección 5 concluye.

\section{PRINCIPALES MEDIDAS DE RIESGO DE CREDITO}

Habitualmente, las medidas de riesgo de crédito consideran indicadores elaborados a partir de información contable. En este sentido, las medidas más utilizadas para evaluar el riesgo de la cartera de colocaciones son el gasto en provisiones, el stock de cartera vencida y los castigos; todos expresados como porcentaje del total de colocaciones ${ }^{2}$.

El gasto en provisiones es una medida basada en pérdidas esperadas dado que corresponde al reconocimiento ex-ante por parte de los bancos de las pérdidas asociadas a la materialización del evento de no pago por parte de los deudores. En términos contables, el gasto en provisiones significa una disminución del patrimonio en conjunto con un aumento del pasivo de los bancos debido al incremento del stock de provisiones. La utilización de este indicador como medida de riesgo de crédito en Chile es amplia. Jara (2005) estima un modelo de riesgo de crédito para 16 bancos nacionales durante el período 1989 a 2004 y relaciona la evolución del gasto en provisiones totales con variables que caracterizan el ciclo económico, controlando además por la heterogeneidad de la industria bancaria. Alfaro, Calvo y Oda (2009) se concentran en el riesgo de crédito de la banca de consumo y relacionan el gasto en

2 No obstante, la distribución de colocaciones por sector económico, el crecimiento del crédito bancario, la exposición de los mayores deudores individuales/consolidados y la exposición a deudores relacionados con la propiedad de una institución bancaria son otros indicadores de riesgo de crédito complementarios considerados dentro de este ámbito (Matus, 2007). 
provisiones de consumo durante el período 1992 a 2009 con variables macroeconómicas como la brecha de producto, la tasa de captación en UF entre 1 a 3 años y la tasa de desempleo. Adicionalmente, Jara, Luna y Oda (2007) y Alfaro y Sagner (2011) señalan que el Banco Central de Chile utiliza el gasto en provisiones desagregado por tipo de cartera como medida de riesgo de crédito en la preparación de las pruebas de tensión o stress test sobre el sistema bancario nacional.

Por otra parte, la cartera vencida o non-performing loans se define de acuerdo al FMI como aquellos créditos cuyos pagos de interés y/o capital se encuentran atrasados por un período de 90 días o más; los pagos por intereses de los últimos 90 días que se encuentran capitalizados, refinanciados o retrasados por acuerdo; o los pagos que se encuentran menos de 90 días atrasados, pero existen buenas razones -como declaración de quiebra del deudor- para dudar que ellos se efectúen en su totalidad $^{3}$. De materializarse alguno de estos eventos, la legislación chilena permite que la totalidad del crédito sea traspasado a cartera vencida en la medida que los bancos hagan efectivas las cláusulas de aceleración pactadas en el contrato. Si bien la Superintendencia de Bancos e Instituciones Financieras (SBIF) publica mensualmente este indicador desde enero de 1997, su utilización en Chile como medida de riesgo de crédito es prácticamente nula, hecho que contrasta con la tendencia observada a nivel internacional ${ }^{4}$.

Por último, los castigos corresponden a aquellos créditos que son eliminados del balance cuando los bancos consideran que ellos son irrecuperables o bien han transcurrido entre 2 a 3 años desde que ingresaron a cartera vencida. En consecuencia, este indicador es una medida rezagada del riesgo de crédito. Contablemente, los castigos reducen el stock de colocaciones y el stock de provisiones, pero no alteran los resultados debido a que ellos se constituyen en conjunto con las provisiones. Al igual que para el caso del gasto en provisiones, Alfaro, Calvo y Oda (2009) utilizan esta medida de riesgo de crédito para la banca de consumo chilena y estudian su dinámica a partir de la evolución de un conjunto de variables que caracterizan el ciclo económico.

Teniendo en cuenta las definiciones anteriores, es posible identificar al menos tres ventajas que señalarían a la cartera vencida como un indicador más apropiado, respecto del gasto en provisiones, para medir el riesgo de crédito agregado e individual del sistema bancario chileno.

En primer lugar, las series de cartera vencida por tipo de crédito poseen una mayor cobertura temporal en comparación con sus homólogas asociadas al gasto en provisiones. Los cambios en la normativa de provisiones introducidos en enero de 2004 consideraron una nueva segmentación del gasto en provisiones totales en consumo, comerciales e hipotecarias, mientras que la cartera vencida ya poseía esta segmentación desde enero de $1997^{5}$.

\footnotetext{
Ver, por ejemplo, Bloem y Freeman (2005).

Ver, por ejemplo, Cortavarria et al. (2000).

Para mayor detalle de este y otros cambios en la normativa chilena de provisiones, ver Adasme, Majnoni y Uribe (2006).
} 
En segundo lugar, y si bien el gasto en provisiones posee una orientación de clasificación de riesgo forward-looking debido a su constitución en base a pérdidas esperadas, dicha orientación es válida sólo a partir del año 2004. Las normas vigentes hasta antes de esta fecha establecían que las provisiones totales debían constituirse una vez que ocurría el evento de no pago, es decir, ellas debían constituirse de acuerdo a una orientación backward-looking.

Por último, el gasto en provisiones corresponde a una medida de riesgo de crédito de carácter discrecional. El cambio en la normativa de provisiones de enero de 2004 facultó a los bancos el desarrollo de modelos de evaluación de riesgo propios como la principal herramienta para estimar, desde el punto de vista del banco evaluador y su aversión relativa al riesgo, las pérdidas esperadas de su cartera de colocaciones. Por el contrario, la definición de cartera vencida asocia el riesgo de crédito con plazos máximos de no pago (generalmente específicos), que pueden ser relacionados con la evolución histórica de variables macroeconómicas que caracterizan el ciclo económico y que, por lo tanto, facilitarían la comparación internacional de los indicadores de riesgo de crédito ${ }^{6}$.

\section{EL INFLUJO DE CARTERA VENCIDA}

En esta sección se define, desde un punto de vista contable, el influjo de cartera vencida. Luego, las propiedades estadísticas de este indicador son comparadas con aquellas del gasto en provisiones como una forma de reforzar su utilización como medida de riesgo de crédito para la industria bancaria nacional. Finalmente, se plantea un modelo econométrico que busca explicar la evolución de esta medida respecto de un conjunto de variables macroeconómicas de la economía chilena.

\subsection{Construcción del Influjo de Cartera Vencida}

Por definición contable, el stock de cartera vencida en el período $t, C V_{t}$, se encuentra conformado por el stock de cartera vencida del período anterior $C V_{t-1}$, el flujo de entrada de cartera vencida $F C V_{t}$ y los castigos $C A_{t}$ (ver Figura 1), ambos en el período $t$, de acuerdo a:

$$
C V_{t}=C V_{t-1}+F C V_{t}-C A_{t}
$$

6 No obstante lo anterior, Matus (2007) advierte que ciertas diferencias en los marcos regulatorios de los países tenderían a dificultar la comparación internacional de los indicadores de riesgo de crédito basados en cartera vencida. En países como Perú o Ecuador, por ejemplo, los plazos para traspasar un crédito a cartera vencida son inferiores, dependiendo del tipo de cartera de colocaciones bajo análisis. 


\section{FIGURA 1}

ESQUEMA

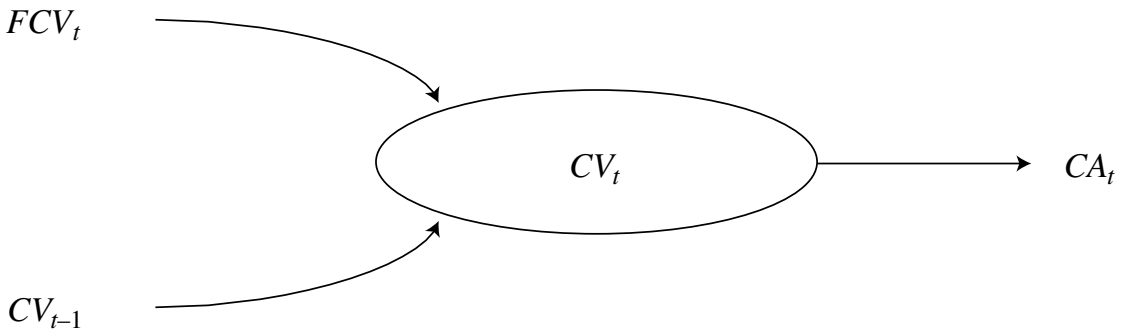

La relación anterior supone, al igual que Alfaro, Calvo y Oda (2009), que las recuperaciones de castigos son despreciables. Así, despejando $F C V_{t}$ desde (1) y normalizando por el stock de colocaciones $L_{t}$ se tiene que:

$$
\frac{F C V_{t}}{L_{t}}=\frac{\Delta C V_{t}+C A_{t}}{L_{t}}
$$

donde $\Delta C V_{t}$ es la variación del stock de cartera vencida en el período $t$. De esta forma, se define el influjo de cartera vencida como $I C V_{t} \equiv\left(F C V_{t} / L_{t}\right)$, el cual es simplemente la variación del stock de cartera vencida ajustada por castigos y normalizada por colocaciones:

$$
I C V_{t}=\frac{\Delta C V_{t}+C A_{t}}{L_{t}}
$$

\subsection{Propiedades Estadísticas del Influjo de Cartera Vencida}

El influjo de cartera vencida fue elaborado a partir de la ecuación (2) para la cartera total, consumo, comercial e hipotecaria empleando datos de la SBIF. La Figura 2 muestra la evolución de la media móvil a 12 meses de las series obtenidas durante el período enero 1998 a junio 2010, mientras que la Tabla 1 reporta los principales estadísticos descriptivos de las series originales. En ambos casos las series son comparadas con el gasto en provisiones normalizado por colocaciones $(G P R)$.

A partir de los resultados anteriores es posible notar cuatro aspectos claves relacionados con ambas medidas de riesgo de crédito. Primero, los índices $I C V$ y GPR exhiben los mayores valores en la cartera de consumo, mientras que los menores valores son observados en la cartera hipotecaria. Durante el período enero 2004 a junio 2010, 
el influjo de cartera vencida y el gasto en provisiones de consumo registraron un valor promedio en torno a 5,7\% y 6,3\%, respectivamente, lo que de acuerdo a Matus et al. (2010) se explicaría por la mayor orientación hacia segmentos de menores ingresos por parte de las divisiones de consumo de las entidades bancarias. Los índices $I C V$ y $G P R$ hipotecario mostraron valores promedio en torno a $0,6 \%$ y $0,4 \%$ durante igual lapso de tiempo, respectivamente, lo que se debería al hecho de que una gran parte de los créditos hipotecarios cuentan con una garantía real que cubre más del 100\% del monto otorgado ${ }^{7}$.

\section{TABLA 1}

ESTADISTICA DESCRIPTIVA ICV / [GPR]

(Porcentaje de las colocaciones)

\begin{tabular}{|c|c|c|c|c|}
\hline & \multicolumn{2}{|c|}{ Total } & \multicolumn{2}{|c|}{ Consumo } \\
\hline & 2004m1-2010m6 & 1997m1-2010m6 & 2004m1-2010m6 & 1997m1-2010m6 \\
\hline Media & $\begin{array}{c}1,364 \\
{[1,607]}\end{array}$ & 1,509 & $\begin{array}{c}5,710 \\
{[6,270]}\end{array}$ & 5,633 \\
\hline Desv. Est. & $\begin{array}{c}0,465 \\
{[0,462]}\end{array}$ & 0,529 & $\begin{array}{c}1,750 \\
{[2,001]}\end{array}$ & 1,681 \\
\hline P10 & $\begin{array}{c}0,902 \\
{[1,102]}\end{array}$ & 0,912 & $\begin{array}{c}3,890 \\
{[3,567]}\end{array}$ & 3,980 \\
\hline P50 & $\begin{array}{c}1,335 \\
{[1,531]}\end{array}$ & 1,415 & $\begin{array}{c}5,444 \\
{[6,010]}\end{array}$ & 5,423 \\
\hline \multirow[t]{3}{*}{ P90 } & $\begin{array}{c}1,840 \\
{[2,403]}\end{array}$ & 2,239 & $\begin{array}{c}8,196 \\
{[9,190]}\end{array}$ & 7,354 \\
\hline & \multicolumn{2}{|c|}{ Comercial } & \multicolumn{2}{|c|}{ Hipotecaria } \\
\hline & 2004m1-2010m6 & 1997m1-2010m6 & 2004m1-2010m6 & 1997m1-2010m6 \\
\hline Media & $\begin{array}{c}0,689 \\
{[1,019]}\end{array}$ & 1,010 & $\begin{array}{c}0,589 \\
{[0,389]}\end{array}$ & 0,512 \\
\hline Desv. Est. & $\begin{array}{c}0,390 \\
{[0,442]}\end{array}$ & 0,659 & $\begin{array}{c}0,413 \\
{[0,300]}\end{array}$ & 0,370 \\
\hline P10 & $\begin{array}{c}0,212 \\
{[0,564]}\end{array}$ & 0,337 & $\begin{array}{c}0,119 \\
{[0,209]}\end{array}$ & 0,116 \\
\hline P50 & $\begin{array}{c}0,657 \\
{[0,964]}\end{array}$ & 0,867 & $\begin{array}{c}0,524 \\
{[0,338]}\end{array}$ & 0,427 \\
\hline P90 & $\begin{array}{c}1,146 \\
{[1,531]}\end{array}$ & 1,932 & $\begin{array}{c}1,175 \\
{[0,551]}\end{array}$ & 0,943 \\
\hline
\end{tabular}

Nota: Todos los índices se encuentran expresados sobre una base anual.

7 Matus et al. (2010) señalan que la normativa vigente hasta antes de octubre de 2009 establecía que los préstamos con letras de crédito y mutuos endosables podían financiar hasta un $75 \%$ y un $80 \%$ del activo inmobiliario, respectivamente. La nueva normativa, por el contrario, permite que los préstamos con letras de crédito pueden financiar hasta un $100 \%$ del valor de la propiedad. 


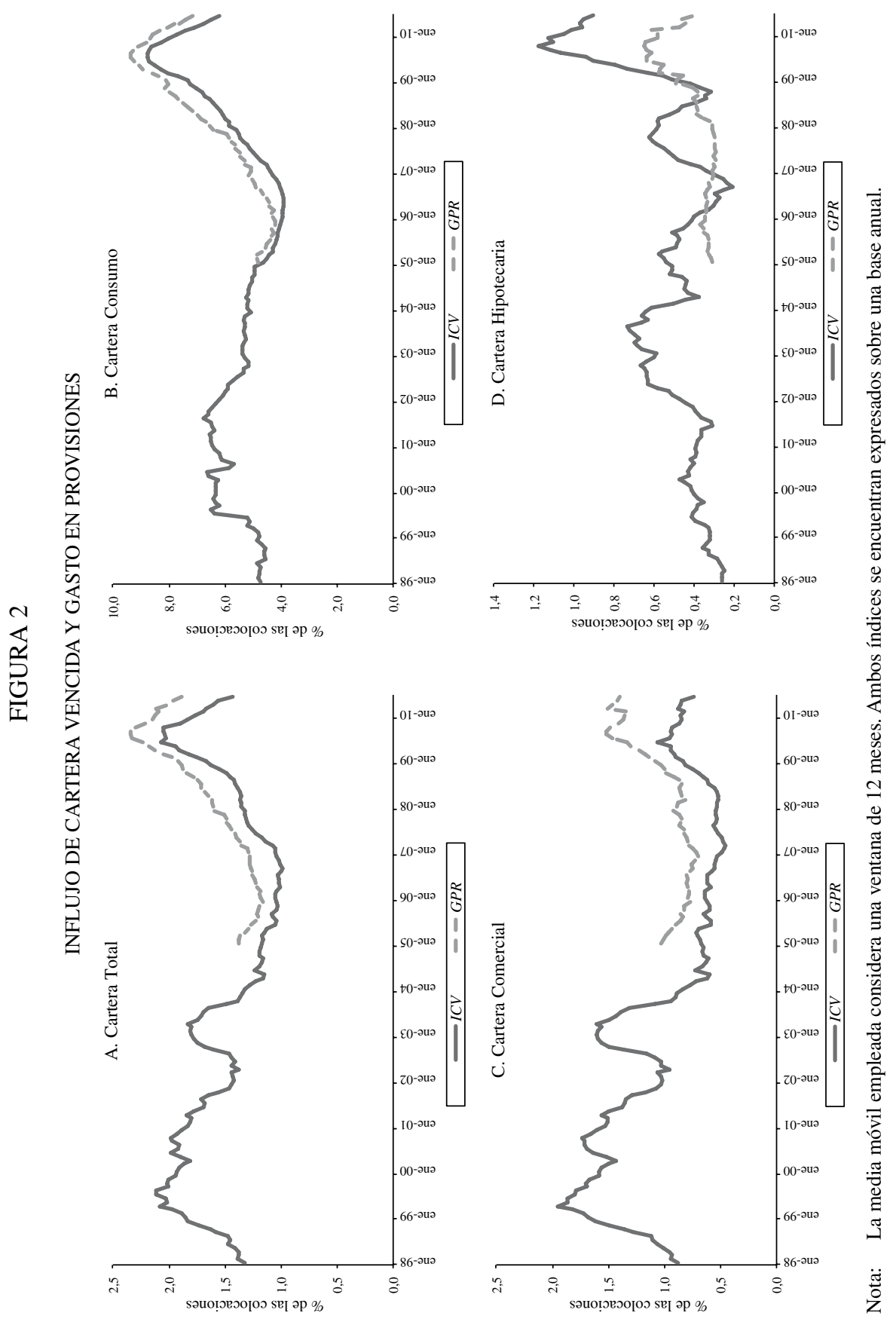


En segundo lugar, el influjo de cartera vencida genera series más suaves para la cartera de consumo e hipotecaria en comparación con el índice de gasto en provisiones (ver Tabla 2). El intervalo al 95\% de confianza asociado a ambas carteras indica que la variabilidad del $I C V$ es estadísticamente menor que aquella observada en GPR. No obstante lo anterior, los resultados de la Tabla 2 revelan que la variabilidad entre ambas medidas de riesgo de crédito no es estadísticamente distinta para el caso de las colocaciones comerciales y totales ${ }^{8}$.

\section{TABLA 2}

COMPARACION DE LA DISPERSION ENTRE ICV Y GPR 2004m1-2010m6

\begin{tabular}{|lcc|}
\hline \multirow{2}{*}{ Cartera } & \multicolumn{2}{c|}{ Coeficiente de Variación de Pearson } \\
\cline { 2 - 3 } Total & $I C V$ & $G P R$ \\
\multirow{2}{*}{ Consumo } & $\mathbf{0 , 1 6 8}$ & $\mathbf{0 , 0 9 7}$ \\
& {$[\mathbf{0 , 0 8 5} ; \mathbf{0 , 2 5 2}]$} & {$[\mathbf{0 , 0 6 2} ; \mathbf{0 , 1 3 3}]$} \\
Comercial & 0,029 & 0,113 \\
& {$[0,016 ; 0,042]$} & {$[0,056 ; 0,171]$} \\
Hipotecaria & 0,539 & 0,279 \\
& {$[0,323 ; 0,755]$} & {$[0,189 ; 0,368]$} \\
& 0,377 & 1,035 \\
\end{tabular}

Nota: $\quad$ El coeficiente de variación de Pearson (CP) se define como $C P=\sigma / \mu$, donde $\sigma$ es la desviación estándar y $\mu$ la media. El intervalo al $95 \%$ de confianza de $C P$ fue calculado mediante el procedimiento sieve bootstrap de Bühlmann (1997) y se reporta en corchetes.

Tercero, la estructura dinámica del influjo de cartera vencida de consumo y total no difiere de forma significativa respecto de aquella del gasto en provisiones. Para las colocaciones comerciales e hipotecarias, por el contrario, los datos señalan diferencias estadísticamente significativas en la dinámica conjunta y timing de dichas series. La Figura 3 muestra la correlación entre el influjo de cartera vencida y adelantos y rezagos del gasto en provisiones para las distintas carteras analizadas 9 . De ella se observa que: (i) $I C V$ y GPR de consumo presentan la mayor correlación para valores contemporáneos de ambos indicadores $\left(\rho_{I C V_{t}, G P R_{t}}=0,855\right)$, siendo el resto de las correlaciones estadísticamente significativas al 5\%; (ii) el GPR comercial

8 La semejanza en el comportamiento de la cartera comercial y total se debe al hecho de que la primera representó cerca del 60\% de las colocaciones totales del sistema entre enero de 2004 y junio de 2010.

9 Dado el tamaño de muestra empleado, las correlaciones son estadísticamente significativas al 5\% para valores, en valor absoluto, mayores que 0,2 . 


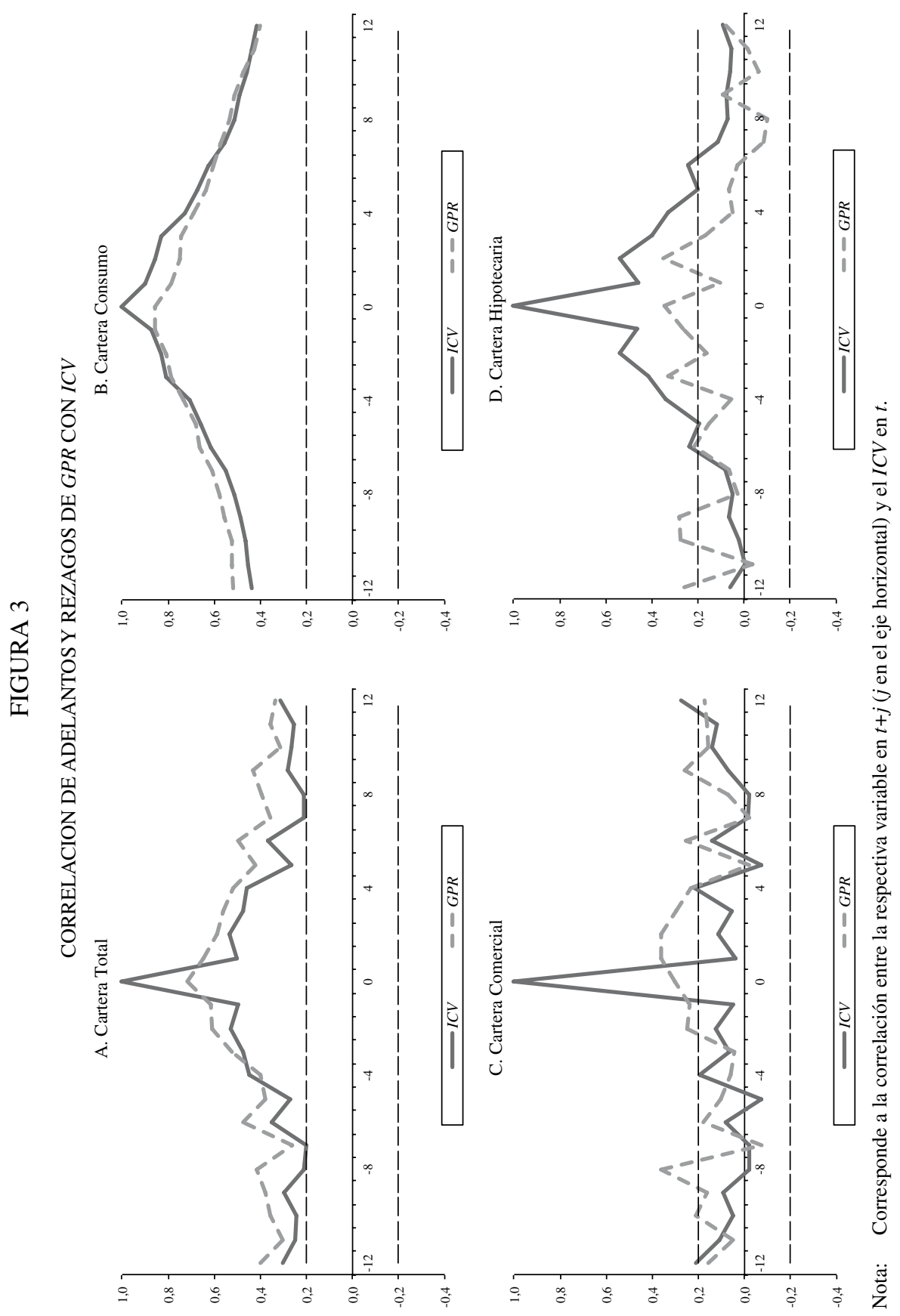


adelanta en 8 meses al $I C V$, dado que la mayor correlación entre ambas series ocurre en $j=-8\left(\rho_{I C V_{t}, G P R_{t-8}}=0,362\right)$; (iii) el $I C V$ hipotecario adelanta en 2 meses al GPR $\left(\rho_{I C V_{t}, G P R_{t+2}}=0,354\right)$, pero al igual que para el caso de la cartera comercial, muchas de las correlaciones encontradas son virtualmente no significativas; y (iv) la mayor correlación entre $I C V$ y $G P R$ de las colocaciones totales ocurre de forma contemporánea $\left(\rho_{I C V_{t}, G P R_{t}}=0,717\right)$, mientras que gran parte de las correlaciones restantes son significativas al $5 \%$.

Finalmente, el influjo de cartera vencida y el gasto en provisiones son estacionarias dentro del período bajo análisis. Los resultados de la Tabla 3 indican que en ambas series, y para todas las carteras consideradas, la hipótesis nula de raíz unitaria es rechazada para niveles de significancia estándares. Este aspecto indicaría que la materialización de shocks transitorios no posee efectos permanentes sobre el riesgo de crédito, y además, los cambios en la normativa chilena no han introducido quiebres estructurales importantes en ambos indicadores.

TABLA 3

TEST DE RAICES UNITARIAS SOBRE ICVY GPR

\begin{tabular}{|lccccc|}
\hline & \multicolumn{2}{c}{$A D F$} & & \multicolumn{2}{c|}{$P P$} \\
\cline { 2 - 3 } \cline { 5 - 6 } ICV & $t$-value & $p$-value & & $t$-value & $p$-value \\
Total & & & & \\
$\quad$ Consumo & $\mathbf{- 7 , 7 2 8}$ & $\mathbf{0 , 0 0 0}$ & & $\mathbf{- 7 , 9 5 9}$ & $\mathbf{0 , 0 0 0}$ \\
Comercial & $-6,894$ & 0,000 & & $-7,008$ & 0,000 \\
Hipotecaria & $-7,636$ & 0,000 & & $-7,826$ & 0,000 \\
GPR & $-7,617$ & 0,000 & & $-7,899$ & 0,000 \\
Total & & & & \\
Consumo & & & & \\
Comercial & $-3,926$ & $\mathbf{0 , 0 0 2}$ & & $-\mathbf{3 , 7 7 9}$ & $\mathbf{0 , 0 0 3}$ \\
Hipotecaria & $-3,303$ & 0,015 & & $-2,956$ & 0,039 \\
& $-9,224$ & 0,000 & & $-5,958$ & 0,000 \\
& & 0,000 & & $-9,209$ & 0,000 \\
\hline
\end{tabular}

Nota: Los tests considerados corresponden a Dickey-Fuller Aumentado $(A D F)$ y Phillips-Perron $(P P)$.

Con todo, el análisis realizado sobre las propiedades estadísticas del influjo de cartera vencida y el gasto en provisiones revelaría ciertas ventajas del primero, a considerar al momento de elegir una medida como proxy del riesgo de crédito para la industria bancaria nacional. En resumen: (i) $I C V$ posee una mayor cobertura temporal respecto de GPR ( 7 años, lo que equivale a un total de 84 observaciones mensuales 
adicionales), aspecto que permite un análisis del riesgo de crédito más robusto debido a que el influjo de cartera vencida considera ciclos económicos expansivos y contractivos que no son capturados muestralmente por el gasto en provisiones; (ii) ICV genera series más suaves que GPR para la cartera de consumo e hipotecaria, hecho que corresponde a una característica deseable al momento de relacionar el riesgo de crédito con la evolución de variables macroeconómicas relevantes; y (iii) la estructura dinámica de $I C V$ no es muy distinta de aquella observada en $G P R$, lo que reflejaría el hecho de que los bancos reconocen el riesgo asociado a su cartera de colocaciones de forma similar bajo ambas definiciones de riesgo de crédito.

\subsection{Modelo Econométrico para el Influjo de Cartera Vencida}

En esta sección se presenta el modelo econométrico empleado para analizar la dinámica del influjo de cartera vencida en base a la evolución de un conjunto de agregados macroeconómicos. En particular, se estimó la siguiente ecuación para la cartera de consumo (con), comercial (com) e hipotecaria (hip):

$$
I C V_{t}^{i}=\alpha_{i}+\sum_{j=1}^{p} \phi_{i, j} I C V_{t-j}^{i}+\sum_{k=0}^{q} \gamma_{i, k}^{\prime} X_{t-k}+\varepsilon_{t}^{i}, \quad i \in\{\text { con,com, hip }\}
$$

donde $I C V_{t-j}^{i}$ es el $j$-ésimo rezago del influjo de cartera vencida de la cartera $i$, y $X_{t-k}$ es un vector que contiene valores contemporáneos y rezagos de variables macroeconómicas.

La inclusión de rezagos de $I C V$ en el modelo econométrico capturaría los efectos provocados por traspasos a cartera vencida y/o constitución de castigos pasados sobre el nivel actual del indicador, es decir, el grado de persistencia presente en la serie. Moody's (2003) sugiere que el traspaso de créditos a cartera vencida luego que ellos se encuentren impagos por un período de 90 días o más se relacionaría con una mayor persistencia. En este sentido, Alfaro, Calvo y Oda (2009) encuentran una componente autorregresiva de orden 1 en su modelo para el gasto en provisiones de consumo, resultado que apoyaría una especificación con rezagos de la variable dependiente, dado que la estructura dinámica de $G P R$ no es muy distinta de aquella observada en $I C V$, como se vio en la sección anterior.

La incorporación de valores contemporáneos y pasados de los agregados macroeconómicos se desprende directamente de la definición de cartera vencida y castigos. Como los primeros corresponden a créditos impagos por 90 días o más, los efectos más notorios de las variables macroeconómicas sobre el influjo de cartera vencida ocurrirían para valores rezagados en 3 meses o más. Adicionalmente, los créditos pueden ser traspasados antes de 90 días a cartera vencida si los bancos consideran que los pagos poseen una alta probabilidad de no pago por parte de los deudores y/o 
ellos pueden ser castigados cuando se consideran totalmente irrecuperables. Así, la inclusión de valores contemporáneos de los agregados macroeconómicos capturaría este segundo efecto sobre $I C V$.

El conjunto de variables macroeconómicas consideradas en este artículo y los respectivos efectos sobre el influjo de cartera vencida son los siguientes ${ }^{10}$ : (i) variación anual del IMACEC ( $\left.g^{I M A C E C}\right)$, donde se espera una relación inversa debido a que un mayor crecimiento económico señalaría una mejora en la capacidad de pago de los deudores y, por lo tanto, tendería a disminuir el riesgo de crédito $^{11}$; (ii) tasa de desempleo (unemp), donde se espera un efecto positivo puesto que el incremento de esta variable significaría una mayor incertidumbre relacionada con el ingreso futuro $\mathrm{y}$, consecuentemente, un aumento del riesgo de crédito (Rinaldi y Sanchis-Arellano, 2006); (iii) variación anual del IPC ( $\left.g^{I P C}\right)$, donde se espera un efecto positivo dado que una mayor tasa de inflación se traduciría en una disminución del ingreso disponible (impuesto inflación); (iv) tasa de interés de captación entre 1 a 3 años (cap) y tasa de mutuos hipotecarios endosables (mhe) como proxies para las tasas de interés de corto y largo plazo, respectivamente, donde se espera una relación inversa debido a que aumentos de dichas variables restringirían el acceso de deudores más riesgosos al mercado del crédito; (v) variación mensual del IPSA ( $\left.g^{I P S A}\right)$, donde se espera una relación inversa con el influjo de cartera vencida comercial en vista que aumentos de esta variable señalarían una menor fragilidad financiera de las empresas y, por ende, un menor riesgo de crédito $^{12}$; (vi) variación mensual del tipo de cambio peso-dólar $\left(g^{\text {dolar }}\right)$, donde se espera un efecto positivo sobre el riesgo de la cartera comercial dado que apreciaciones del dólar aumentarían, vía efecto precio, la exposición de empresas en deudas denominadas en esta moneda e incrementarían su riesgo de no pago; y (vii) sorpresas en el crecimiento de las colocaciones $\left(g^{c o l}-\hat{g}^{\text {col }}\right)$, las cuales fueron aproximadas como la diferencia entre la tasa de crecimiento mensual efectiva de las colocaciones y la tasa de crecimiento estimada a partir de un proceso $\operatorname{AR}(1)$, y donde se espera un efecto positivo debido a que una colocación de créditos por sobre niveles "esperados" se traduciría en un mayor riesgo de crédito (Schularick y Taylor, 2009; Borio y Drehmann, 2009).

\section{RESULTADOS}

En esta sección se presentan los resultados correspondientes a la estimación del modelo econométrico para el influjo de cartera vencida de consumo, comercial e hipotecario. Finalmente, las especificaciones identificadas como favoritas son sometidas a

10 Para mayor detalle de las variables consideradas y sus fuentes, ver el Anexo 1.

11 En este sentido, Alfaro, Gallardo y Stein (2010) encuentran una relación inversa y estadísticamente significativa entre el ingreso y la probabilidad de no pago de la deuda de consumo e hipotecaria de los hogares chilenos.

12 Shu (2002) indica que la variación del precio de activos es frecuentemente citada en la literatura como un indicador líder de las condiciones económicas. 
un ejercicio de proyección fuera de muestra como una forma de evaluar su capacidad predictiva dentro de distintos horizontes de proyección.

\subsection{Modelo Econométrico}

Los resultados luego de estimar distintas versiones de la ecuación (3) para cada cartera de colocaciones se presentan en las Tablas 4 a 6 . En ellas, las tres primeras columnas difieren sólo en la especificación de la componente autorregresiva de $I C V$, mientras que las tres últimas columnas consideran rezagos tanto del influjo de cartera vencida como de los agregados macroeconómicos.

Las estimaciones para la cartera de consumo (Tabla 4) muestran que dicha serie posee un cierto grado de persistencia en su dinámica de ajuste, aspecto que se deriva de la significancia estadística asociada al primer y tercer rezago de $I C V$. La magnitud de los coeficientes estimados indica que ante un aumento inesperado de $1 \%$ en el riesgo de crédito de la cartera de consumo, cerca del $96 \%$ de este shock es disipado en un horizonte no mayor a 1 año. En adición, el intervalo al 95\% de confianza sitúa el valor de largo plazo de la serie entre 5,33\% y 5,94\% anual, resultados que son consistentes con la estadística descriptiva presentada en la Tabla $1^{13}$. Por el lado de los agregados macroeconómicos, la variación anual del IMACEC posee efectos negativos y significativos, tanto para su valor contemporáneo como para su rezago a 3 meses: un aumento de $1 \%$ en el crecimiento económico se traduciría en una disminución del $I C V$ de consumo entre $0,10 \%$ y $0,25 \%$ en el mismo mes, y entre $0,03 \%$ y $0,21 \%$ luego de 3 meses. Las diferencias en las magnitudes estimadas revelarían una mayor sensibilidad de esta medida de riesgo de crédito a la mayor (menor) constitución de cartera vencida en aquellos créditos de consumo con una mora menor a 90 días y bajo escenarios de contracción (expansión) económica. Por el contrario, la tasa de desempleo -si bien presenta los signos esperados- no posee efectos significativos sobre el ICV, resultado que se explicaría por la naturaleza backward-looking del influjo de cartera vencida en contraste con la relación desempleo a ingreso futuro postulada por Rinaldi y Sanchis-Arellano (2006). Respecto de la inflación anual, ella posee una relación positiva y estadísticamente significativa con el $I C V$ de consumo, donde los mayores efectos son observados con un rezago de 3 meses. En este sentido, un aumento de $1 \%$ en la inflación anual ocasionaría un incremento entre $0,13 \%$ y $0,31 \%$ en el riesgo de crédito de esta cartera en los 3 meses posteriores, es decir, al momento de traspasar créditos de consumo atrasados en 90 días o más a cartera vencida. La tasa de interés de corto plazo, por su parte, presenta una relación contemporánea inversa con el ICV de consumo, lo cual se atribuiría a la menor (mayor) entrada de clientes riesgosos al mercado del crédito ante tasas de interés altas (bajas).

13 El valor de largo plazo del influjo de cartera vencida ( $\operatorname{CV}_{L P}^{i}$ ) fue calculado como $I C V_{L P}^{i}=\left(\hat{\alpha}_{i}+\sum_{k=0}^{q} \hat{\gamma}_{i, k}^{\prime} \bar{X}_{t-k}\right) /\left(1-\sum_{j=1}^{p} \hat{\phi}_{i, j}\right), \quad i \in\{$ con,com,hip $\}$, donde $\bar{X}_{t-k}^{L P}$ corresponde al valor promedio de las variables macroeconómicas consideradas. 
TABLA 4

RESULTADOS ESTIMACIONES MODELO ICV CARTERA DE CONSUMO

\begin{tabular}{|c|c|c|c|c|c|c|}
\hline & (1) & (2) & (3) & (4) & (5) & (6) \\
\hline$I C V_{t-1}$ & & $\begin{array}{c}0,399 \\
{[3,05]^{* * *}}\end{array}$ & $\begin{array}{c}0,309 \\
{[3,85] * * *}\end{array}$ & & $\begin{array}{c}0,377 \\
{[2,97]^{* * *}}\end{array}$ & $\begin{array}{c}0,342 \\
{[3,86] * * *}\end{array}$ \\
\hline$I C V_{t-3}$ & & & $\begin{array}{c}0,332 \\
{[5,31]^{* * *}}\end{array}$ & & & $\begin{array}{c}0,321 \\
{[4,09] * * *}\end{array}$ \\
\hline$g_{t}^{I M A C E C}$ & $\begin{array}{c}-0,249 \\
{[5,45]^{* * *}}\end{array}$ & $\begin{array}{c}-0,142 \\
{[3,37] * * *}\end{array}$ & $\begin{array}{c}-0,099 \\
{[2,86] * * *}\end{array}$ & & & \\
\hline$g_{t-3}^{I M A C E C}$ & & & & $\begin{array}{c}-0,209 \\
{[5,13]^{* * *}}\end{array}$ & $\begin{array}{c}-0,107 \\
{[2,51]^{* * *}}\end{array}$ & $\begin{array}{c}-0,028 \\
{[0,65]}\end{array}$ \\
\hline unemp $_{t}$ & $\begin{array}{l}0,013 \\
{[0,17]}\end{array}$ & $\begin{array}{l}0,037 \\
{[0,47]}\end{array}$ & $\begin{array}{l}0,062 \\
{[0,92]}\end{array}$ & & & \\
\hline unemp $_{t-3}$ & & & & $\begin{array}{l}0,024 \\
{[0,32]}\end{array}$ & $\begin{array}{l}0,009 \\
{[0,13]}\end{array}$ & $\begin{array}{l}0,025 \\
{[0,38]}\end{array}$ \\
\hline$g_{t}^{I P C}$ & $\begin{array}{c}0,217 \\
{[5,24]^{* * *}}\end{array}$ & $\begin{array}{c}0,148 \\
{[3,53]^{* * *}}\end{array}$ & $\begin{array}{c}0,133 \\
{[3,81]^{* * *}}\end{array}$ & & & \\
\hline$g_{t-3}^{I P C}$ & & & & $\begin{array}{c}0,307 \\
{[6,13]^{* * *}}\end{array}$ & $\begin{array}{c}0,195 \\
{[3,62]^{* * *}}\end{array}$ & $\begin{array}{c}0,127 \\
{[2,81] * * *}\end{array}$ \\
\hline $\operatorname{cap}_{t}$ & $\begin{array}{c}-0,089 \\
{[3,10]^{* * *}}\end{array}$ & $\begin{array}{c}-0,061 \\
{[1,99]^{* *}}\end{array}$ & $\begin{array}{c}0,055 \\
{[2,23]^{* *}}\end{array}$ & & & \\
\hline $\operatorname{cap}_{t-3}$ & & & & $\begin{array}{c}-0,071 \\
{[2,79]^{* * *}}\end{array}$ & $\begin{array}{c}-0,054 \\
{[1,89]^{* *}}\end{array}$ & $\begin{array}{r}-0,039 \\
{[1,63]}\end{array}$ \\
\hline$g_{t}^{c o l}-\hat{g}_{t}^{c o l}$ & $\begin{array}{l}0,012 \\
{[0,61]}\end{array}$ & $\begin{array}{l}0,004 \\
{[0,02]}\end{array}$ & $\begin{array}{l}0,014 \\
{[1,21]}\end{array}$ & & & \\
\hline$g_{t-3}^{c o l}-\hat{g}_{t-3}^{c o l}$ & & & & $\begin{array}{c}0,032 \\
{[3,06]^{* * *}}\end{array}$ & $\begin{array}{c}0,028 \\
{[2,82]^{* * *}}\end{array}$ & $\begin{array}{c}0,028 \\
{[2,59] * * *}\end{array}$ \\
\hline$D^{T 2}$ & $\begin{array}{c}-0,545 \\
{[1,76]^{*}}\end{array}$ & $\begin{array}{r}-0,376 \\
{[1,38]}\end{array}$ & $\begin{array}{c}-0,719 \\
{[2,89] * * *}\end{array}$ & $\begin{array}{c}-0,673 \\
{[2,36]^{* *}}\end{array}$ & $\begin{array}{c}-0,512 \\
{[2,04]^{* *}}\end{array}$ & $\begin{array}{c}-0,712 \\
{[3,05] * * *}\end{array}$ \\
\hline$D^{T 3}$ & $\begin{array}{c}-0,785 \\
{[2,75]^{* * *}}\end{array}$ & $\begin{array}{c}-0,663 \\
{[2,22]^{* *}}\end{array}$ & $\begin{array}{c}-0,719 \\
{[2,79] * * *}\end{array}$ & $\begin{array}{c}-1,137 \\
{[3,58]^{* * *}}\end{array}$ & $\begin{array}{c}-0,950 \\
{[2,69]^{* * *}}\end{array}$ & $\begin{array}{c}-0,970 \\
{[3,21] * * *}\end{array}$ \\
\hline$D^{T 4}$ & $\begin{array}{c}-0,539 \\
{[2,13]^{* *}}\end{array}$ & $\begin{array}{r}-0,253 \\
{[1,03]}\end{array}$ & $\begin{array}{c}-0,427 \\
{[1,98]^{* *}}\end{array}$ & $\begin{array}{c}-0,709 \\
{[2,87]^{* * *}}\end{array}$ & $\begin{array}{r}-0,387 \\
{[1,52]}\end{array}$ & $\begin{array}{c}-0,485 \\
{[2,20]^{* *}}\end{array}$ \\
\hline Constante & $\begin{array}{c}6,977 \\
{[7,14]^{* * *}}\end{array}$ & $\begin{array}{c}4,493 \\
{[3,22] * * *}\end{array}$ & $\begin{array}{c}3,320 \\
{[3,56] * * *}\end{array}$ & $\begin{array}{c}6,086 \\
{[6,40]^{* * *}}\end{array}$ & $\begin{array}{c}4,035 \\
{[3,29]^{* * *}}\end{array}$ & $\begin{array}{c}2,489 \\
{[2,65] * * *}\end{array}$ \\
\hline Obs. & 164 & 163 & 161 & 164 & 163 & 161 \\
\hline$R^{2}$ & 0,480 & 0,588 & 0,688 & 0,506 & 0,599 & 0,680 \\
\hline$R^{2} \mathrm{Adj}$ & 0,450 & 0,561 & 0,665 & 0,477 & 0,573 & 0,657 \\
\hline$A I C$ & 545,10 & 506,89 & 458,34 & 536,77 & 502,17 & 462,42 \\
\hline$B I C$ & 573,00 & 537,83 & 492,24 & 564,67 & 533,11 & 496,31 \\
\hline
\end{tabular}

Nota: Valor absoluto test $t$ obtenido mediante sieve bootstrap en corchetes. *, ** y *** significativos al $10 \%, 5 \%$ y $1 \%$, respectivamente. 
Los resultados asociados a las sorpresas en el crecimiento de las colocaciones muestran que los coeficientes estimados para dicha variable son positivos y económicamente significativos sólo para su rezago a 3 meses. Lo anterior indicaría que el crecimiento de las colocaciones de consumo por sobre niveles "esperados" potenciaría un aumento del riesgo de crédito (en torno al 0,03\% del exceso de crecimiento) como consecuencia de la mayor aprobación de créditos riesgosos y su mayor probabilidad de traspaso a cartera vencida. Por último, y consistente con la estructura de mercado de la deuda de consumo, notamos que el influjo de cartera vencida posee una marcada estacionalidad: el $I C V$ de consumo tiende en promedio a disminuir a partir del segundo trimestre y manifiesta su mayor caída durante el tercer trimestre de cada año (entre $0,66 \%$ y $1,14 \%$ ).

Para el caso de la cartera comercial (Tabla 5), la persistencia predicha por el modelo econométrico es bastante mayor que aquella estimada para las colocaciones de consumo: ante un aumento inesperado de $1 \%$ en el influjo de cartera vencida comercial, poco más de $70 \%$ de la magnitud de este shock es absorbido luego de 1 año, comportamiento que es consecuencia directa de la significancia estadística asociada al rezago a 12 meses de $I C V$. Al igual que para el caso anterior, el valor de largo plazo de la serie es consistente con la estadística descriptiva desplegada en la Tabla 1 y se encuentra, con un 5\% de significancia, en el intervalo 0,82\%-1,20\%. Respecto de las variables macroeconómicas consideradas, sólo la variación anual del IMACEC y la variación mensual del tipo de cambio peso-dólar poseen efectos económicamente significativos sobre el influjo de cartera vencida comercial, aunque el timing asociado a ellos es distinto. En el primer caso, los efectos más notorios se observan luego de 9 meses, o visto de otra forma, 6 meses después de traspasar créditos comerciales impagos en más de 90 días a cartera vencida. Este hecho podría deberse a que, ante escenarios de contracción (expansión) económica, el reconocimiento promedio del mayor (menor) riesgo de crédito se efectuaría sobre las cuotas retrasadas y no sobre el total de la deuda ${ }^{14}$. Notamos, sin embargo, que la magnitud de los coeficientes estimados es pequeña en relación con la cartera de consumo: un aumento del crecimiento económico de $1 \%$ se traduciría en una disminución del ICV comercial entre $0,01 \%$ y $0,04 \%$ en los 9 meses posteriores. En el segundo caso, por el contrario, los efectos más notorios del tipo de cambio peso-dólar sobre el influjo de cartera vencida se observan de forma contemporánea. Así, una apreciación del dólar de $1 \%$ ocasionaría una aumento del $I C V$ comercial entre $0,02 \%$ y $0,04 \%$ durante el mismo mes, lo que se debería a la mayor exposición a este riesgo, vía efecto precio, de deudas adquiridas en esta divisa.

Finalmente, la Tabla 6 muestra los resultados obtenidos para la cartera hipotecaria. De ella notamos que la persistencia de esta medida de riesgo de crédito es muy similar en duración a aquella observada para la deuda de consumo: ante un incremento inesperado del ICV hipotecario, prácticamente la totalidad de este shock se desvanece luego de 12 meses y la serie converge a su valor de largo plazo que, de acuerdo los

14 Matus (2007) argumenta que la regulación chilena para la cartera vencida permite a los bancos traspasar sólo la porción impaga de los préstamos en cuotas, más que la totalidad del crédito, luego de ocurrido el default. 
TABLA 5

RESULTADOS ESTIMACIONES MODELO ICV CARTERA COMERCIAL

\begin{tabular}{|c|c|c|c|c|c|c|}
\hline & (1) & (2) & (3) & (4) & (5) & (6) \\
\hline$I C V_{t-1}$ & & $\begin{array}{c}0,351 \\
{[4,24]^{* * *}}\end{array}$ & $\begin{array}{c}0,295 \\
{[3,51]^{* * *}}\end{array}$ & & $\begin{array}{c}0,355 \\
{[4,09]^{* * *}}\end{array}$ & $\begin{array}{c}0,306 \\
{[3,52]^{* * *}}\end{array}$ \\
\hline$I C V_{t-3}$ & & $\begin{array}{c}0,156 \\
{[1,84]^{*}}\end{array}$ & $\begin{array}{c}0,138 \\
{[1,96]^{*}}\end{array}$ & & $\begin{array}{c}0,179 \\
{[2,08]^{* *}}\end{array}$ & $\begin{array}{c}0,166 \\
{[2,22]^{* *}}\end{array}$ \\
\hline$I C V_{t-12}$ & & & $\begin{array}{c}0,298 \\
{[4,28]^{* * *}}\end{array}$ & & & $\begin{array}{c}2,94 \\
{[3,91]^{* * *}}\end{array}$ \\
\hline$g_{t}^{I M A C E C}$ & $\begin{array}{c}-0,049 \\
{[2,19] * *}\end{array}$ & $\begin{array}{r}-0,019 \\
{[0,92]}\end{array}$ & $\begin{array}{r}-0,026 \\
{[1,31]}\end{array}$ & & & \\
\hline$g_{t-9}^{I M A C E C}$ & & & & $\begin{array}{c}-0,039 \\
{[2,18]^{* *}}\end{array}$ & $\begin{array}{l}-0,026 \\
{[1,71]^{*}}\end{array}$ & $\begin{array}{l}-0,013 \\
{[1,91]^{*}}\end{array}$ \\
\hline unemp $_{t}$ & $\begin{array}{l}0,004 \\
{[0,14]}\end{array}$ & $\begin{array}{l}0,015 \\
{[0,53]}\end{array}$ & $\begin{array}{c}0,062 \\
{[1,83]^{*}}\end{array}$ & & & \\
\hline unemp $_{t-3}$ & & & & $\begin{array}{l}0,042 \\
{[1,38]}\end{array}$ & $\begin{array}{l}0,042 \\
{[1,55]}\end{array}$ & $\begin{array}{c}0,076 \\
{[2,41]^{* *}}\end{array}$ \\
\hline$g_{t}^{I P S A}$ & $\begin{array}{r}-0,007 \\
{[0,84]}\end{array}$ & $\begin{array}{c}-0,008 \\
{[0,99]}\end{array}$ & $\begin{array}{r}-0,007 \\
{[0,88]}\end{array}$ & & & \\
\hline$g_{t-3}^{I P S A}$ & & & & $\begin{array}{r}-0,013 \\
{[1,11]}\end{array}$ & $\begin{array}{r}-0,014 \\
{[1,37]}\end{array}$ & $\begin{array}{l}-0,016 \\
{[1,73]^{*}}\end{array}$ \\
\hline$g_{t}^{\text {dolar }}$ & $\begin{array}{c}0,041 \\
{[2,15]^{* *}}\end{array}$ & $\begin{array}{c}0,025 \\
{[1,68]^{*}}\end{array}$ & $\begin{array}{c}0,023 \\
{[1,69]^{*}}\end{array}$ & & & \\
\hline$g_{t-3}^{\text {dolar }}$ & & & & $\begin{array}{c}0,017 \\
{[1,14]}\end{array}$ & $\begin{array}{l}0,007 \\
{[0,51]}\end{array}$ & $\begin{array}{l}0,008 \\
{[0,63]}\end{array}$ \\
\hline$g_{t}^{c o l}-\hat{g}_{t}^{c o l}$ & $\begin{array}{l}0,008 \\
{[0,16]}\end{array}$ & $\begin{array}{l}0,015 \\
{[0,30]}\end{array}$ & $\begin{array}{l}0,006 \\
{[0,12]}\end{array}$ & & & \\
\hline$g_{t-3}^{c o l}-\hat{g}_{t-3}^{c o l}$ & & & & $\begin{array}{c}0,011 \\
{[2,15]^{* *}}\end{array}$ & $\begin{array}{c}0,075 \\
{[1,44]}\end{array}$ & $\begin{array}{l}0,045 \\
{[0,84]}\end{array}$ \\
\hline Constante & $\begin{array}{c}1,098 \\
{[3,23]^{* * *}}\end{array}$ & $\begin{array}{c}0,681 \\
{[2,02]^{* *}}\end{array}$ & $\begin{array}{c}0,946 \\
{[2,70]^{* * *}}\end{array}$ & $\begin{array}{c}1,532 \\
{[4,49]^{* * *}}\end{array}$ & $\begin{array}{c}0,954 \\
{[3,12]^{* * *}}\end{array}$ & $\begin{array}{c}1,027 \\
{[3,18]^{* * *}}\end{array}$ \\
\hline Obs. & 164 & 161 & 152 & 164 & 161 & 152 \\
\hline$R^{2}$ & 0,276 & 0,434 & 0,510 & 0,256 & 0,445 & 0,519 \\
\hline$R^{2} \mathrm{Adj}$ & 0,233 & 0,392 & 0,468 & 0,212 & 0,404 & 0,478 \\
\hline$A I C$ & 284,94 & 246,92 & 219,89 & 289,38 & 243,57 & 217,13 \\
\hline$B I C$ & 303,54 & 271,57 & 247,11 & 307,98 & 268,22 & 244,34 \\
\hline
\end{tabular}

Nota: Valor absoluto test $t$ obtenido mediante sieve bootstrap en corchetes. *, ** y *** significativos al $10 \%, 5 \%$ y $1 \%$, respectivamente. 
TABLA 6

RESULTADOS ESTIMACIONES MODELO ICV CARTERA HIPOTECARIA

\begin{tabular}{|c|c|c|c|c|c|c|}
\hline & (1) & (2) & (3) & (4) & (5) & (6) \\
\hline$I C V_{t-1}$ & & $\begin{array}{c}0,332 \\
{[3,64] * * *}\end{array}$ & $\begin{array}{c}0,261 \\
{[2,74]^{* * *}}\end{array}$ & & $\begin{array}{c}0,346 \\
{[3,92] * * *}\end{array}$ & $\begin{array}{c}0,278 \\
{[3,08]^{* * *}}\end{array}$ \\
\hline$I C V_{t-2}$ & & & $\begin{array}{c}0,266 \\
{[2,22]^{* *}}\end{array}$ & & & $\begin{array}{c}0,214 \\
{[2,14]^{* *}}\end{array}$ \\
\hline$g_{t}^{I M A C E C}$ & $\begin{array}{c}-0,033 \\
{[3,23]^{* * *}}\end{array}$ & $\begin{array}{l}-0,021 \\
{[1,96]^{*}}\end{array}$ & $\begin{array}{r}-0,015 \\
{[1,54]}\end{array}$ & & & \\
\hline$g_{t-3}^{I M A C E C}$ & & & & $\begin{array}{c}-0,033 \\
{[3,19]^{* * *}}\end{array}$ & $\begin{array}{c}-0,022 \\
{[2,02]^{* *}}\end{array}$ & $\begin{array}{c}-0,016 \\
{[1,64]}\end{array}$ \\
\hline unemp $_{t}$ & $\begin{array}{l}0,015 \\
{[0,94]}\end{array}$ & $\begin{array}{l}0,012 \\
{[0,77]}\end{array}$ & $\begin{array}{l}0,011 \\
{[0,73]}\end{array}$ & & & \\
\hline unemp $_{t-3}$ & & & & $\begin{array}{l}0,010 \\
{[0,64]}\end{array}$ & $\begin{array}{l}0,005 \\
{[0,30]}\end{array}$ & $\begin{array}{c}0,002 \\
{[0,13]}\end{array}$ \\
\hline$g_{t}^{I P C}$ & $\begin{array}{l}0,022 \\
{[1,47]}\end{array}$ & $\begin{array}{l}0,013 \\
{[0,79]}\end{array}$ & $\begin{array}{l}0,006 \\
{[0,39]}\end{array}$ & & & \\
\hline$g_{t-3}^{I P C C}$ & & & & $\begin{array}{l}0,006 \\
{[0,31]}\end{array}$ & $\begin{array}{l}0,002 \\
{[0,11]}\end{array}$ & $\begin{array}{c}0,002 \\
{[0,09]}\end{array}$ \\
\hline mhe $_{t}$ & $\begin{array}{c}-0,061 \\
{[4,09]^{* * *}}\end{array}$ & $\begin{array}{c}-0,043 \\
{[2,86]^{* * *}}\end{array}$ & $\begin{array}{c}-0,035 \\
{[2,33]^{* *}}\end{array}$ & & & \\
\hline$m h e_{t-3}$ & & & & $\begin{array}{c}-0,055 \\
{[3,03]^{* * *}}\end{array}$ & $\begin{array}{c}-0,037 \\
{[2,17]^{* *}}\end{array}$ & $\begin{array}{l}-0,029 \\
{[1,79]^{*}}\end{array}$ \\
\hline$g_{t}^{c o l}-\hat{g}_{t}^{c o l}$ & $\begin{array}{c}0,015 \\
{[2,55]^{* * *}}\end{array}$ & $\begin{array}{c}0,015 \\
{[3,07] * * *}\end{array}$ & $\begin{array}{c}0,016 \\
{[3,17]^{* * *}}\end{array}$ & & & \\
\hline$g_{t-3}^{c o l}-\hat{g}_{t-3}^{c o l}$ & & & & $\begin{array}{c}0,013 \\
{[2,54]^{* * *}}\end{array}$ & $\begin{array}{c}0,009 \\
{[1,98]^{* *}}\end{array}$ & $\begin{array}{l}0,007 \\
{[1,64]}\end{array}$ \\
\hline Constante & $\begin{array}{c}1,237 \\
{[5,25]^{* * *}}\end{array}$ & $\begin{array}{c}0,833 \\
{[2,98]^{* * *}}\end{array}$ & $\begin{array}{c}0,641 \\
{[2,37]^{* *}}\end{array}$ & $\begin{array}{c}1,091 \\
{[5,00]^{* * *}}\end{array}$ & $\begin{array}{c}0,697 \\
{[2,59]^{* * *}}\end{array}$ & $\begin{array}{c}0,510 \\
{[1,85]^{*}}\end{array}$ \\
\hline Obs. & 164 & 163 & 162 & 164 & 163 & 162 \\
\hline$R^{2}$ & 0,238 & 0,329 & 0,362 & 0,177 & 0,278 & 0,309 \\
\hline$R^{2} \mathrm{Adj}$. & 0,214 & 0,303 & 0,333 & 0,150 & 0,251 & 0,277 \\
\hline AIC & 103,90 & 85,61 & 79,48 & 116,58 & 97,36 & 92,56 \\
\hline$B I C$ & 122,50 & 107,27 & 104,18 & 135,18 & 119,01 & 117,26 \\
\hline
\end{tabular}

Nota: Valor absoluto test $t$ obtenido mediante sieve bootstrap en corchetes. *,**y *** significativos al $10 \%, 5 \%$ y $1 \%$, respectivamente. 
coeficientes estimados, se encuentra dentro del intervalo 0,44\%-0,59\%. La variación anual del IMACEC vuelve a aparecer como variable estadísticamente significativa, pero un aspecto interesante que se desprende de los resultados es la virtual simetría entre su efecto contemporáneo y a 3 meses sobre el $I C V$ hipotecario.

Este resultado implicaría que la evolución actual del crecimiento económico es un buen predictor de la capacidad de pago de los hogares tanto en el corto como en el mediano plazo. En cuanto a la tasa de interés de largo plazo, ella posee efectos negativos y económicamente significativos sobre el influjo de cartera vencida hipotecaria: un aumento de $1 \%$ generaría una disminución del riesgo de crédito entre $0,04 \%$ y $0,06 \%$ durante el mismo mes como reflejo de la menor entrada inmediata de clientes más riesgosos al mercado de créditos hipotecarios. Por último, el crecimiento de las colocaciones hipotecarias por sobre niveles "esperados" manifestaría sus mayores efectos de forma contemporánea, donde el riesgo de crédito se incrementaría en torno al $0,02 \%$ de la magnitud de este exceso de crecimiento, resultado que es consistente con Schularick y Taylor (2009), y Borio y Drehmann (2009).

Con todo, y basado en los criterios de información de Akaike (AIC) y Schwarz $(B I C)$, notamos que las especificaciones identificadas como favoritas para la cartera de consumo, comercial e hipotecaria son (3), (6), y (3), respectivamente. Sin embargo, el ajuste global de estas especificaciones -medido por el coeficiente $R^{2}$ ajustadodista mucho de ser homogéneo y en algunos casos es bastante bajo $(67 \%, 48 \%$ y $33 \%$ para las carteras de consumo, comercial e hipotecario, respectivamente), hecho que puede tener importantes implicancias al momento de generar proyecciones de dichas medidas de riesgo.

Es por esta razón que en la próxima sección se realiza un ejercicio de proyección fuera de muestra por tipo de cartera, donde el poder de predicción de las distintas especificaciones estimadas es comparado mediante el test propuesto por Diebold y Mariano (1995). Este ejercicio permitirá identificar, sobre la base de la literatura de proyecciones, aquellas especificaciones que generan las "mejores" proyecciones fuera de muestra para los distintos horizontes de proyección considerados.

\subsection{Proyección Fuera de Muestra}

Sea $\widehat{I C V}_{h, t}^{i}$ la proyección fuera de muestra del influjo de cartera vencida $i \in\{$ con,com, hip $\}$ obtenida a partir de la especificación $h$. A partir de esta definición, el procedimiento empleado para generar las distintas proyecciones de $I C V$ se basó en un simple método de tres pasos. En primer lugar, la ecuación (3) fue estimada para cada cartera $i$ utilizando datos del período enero 1997 a junio 2008, es decir, considerando una ventana de estimación igual a 11,5 años. Luego, la proyección $\widehat{I C V}_{h, t}$ se obtuvo utilizando los parámetros estimados en el paso anterior, en conjunto con los valores observados de los agregados macroeconómicos y los rezagos de $I C V$. Finalmente, el procedimiento se repitió avanzando un mes en cada iteración y manteniendo inalterada la ventana de estimación hasta que la última fecha proyectada corresponda a junio de 2010 . 
Adicionalmente, la proyección del ICV sobre la base de dos especificaciones alternativas requirió definir la función de pérdida (a minimizar) que se utilizará para evaluar la capacidad predictiva de cada una de ellas. En este sentido, la función de pérdida utilizada en este artículo corresponde al Error Cuadrático Medio asociado a la especificación $h$ de la cartera $i\left(E C M_{h}^{i}\right)$, la cual se define como:

$$
E C M_{h}^{i}=\frac{1}{T} \sum_{t=1}^{T} g\left(\widehat{I C V}_{h, t}^{i}, I C V_{t}^{i}\right), \quad i \in\{\text { con,com, hip }\}
$$

donde $g\left(\widehat{I C V}_{h, t}^{i}, I C V_{t}^{i}\right) \equiv\left(I C V_{t}^{i}-\widehat{I C V}_{h, t}^{i}\right)^{2}$ y $T$ es el tamaño de muestra empleado en las proyecciones. De esta forma, la hipótesis nula del test Diebold y Mariano (1995) relacionada con la igualdad de precisión entre las proyecciones generadas por las especificaciones $l$ y $m$ en competencia, $l, m \in h$, es $E\left[d_{l, m}^{i}\right]=0$, donde $d_{l, m}^{i}=g\left(\widehat{I C V}_{l, t}^{i}, I C V_{t}^{i}\right)-g\left(\widehat{I C V}_{m, t}^{i}, I C V_{t}^{i}\right)$ es el diferencial de pérdida para $i \in\{$ con, com, hip $\}$.

Los resultados luego de aplicar el test de Diebold y Mariano sobre las proyecciones a distintos horizontes, obtenidas de las especificaciones estimadas en las Tablas 4 a 6 para cada cartera de colocaciones $i \in\{c o n, c o m, h i p\}$, se muestran en las Tablas 7 a 9. Este ejercicio consideró, además, las proyecciones provenientes de una especificación alternativa (especificación (7)), la cual corresponde a una caminata aleatoria, como una forma de evaluar más ácidamente la capacidad predictiva de las especificaciones bajo análisis ${ }^{15}$.

Los resultados para la cartera de consumo (Tabla 7) señalan que dentro de un horizonte de proyección de hasta 6 meses la caminata aleatoria tiende a generar proyecciones más acertadas que el resto de las especificaciones evaluadas, pero a medida que dicho horizonte es más prolongado la proyección correspondiente a la especificación (3) comienza a surgir como favorita, la cual es a su vez aquella que presenta los menores criterios de información $A I C$ y $B I C^{16}$.

Para el caso de la cartera comercial, los resultados exhibidos en la Tabla 8 son algo más confusos de interpretar. La caminata aleatoria es nuevamente el modelo que genera las proyecciones puntuales más certeras del $I C V$ dentro de un horizonte de hasta un año. No obstante lo anterior, la capacidad predictiva promedio de esta modelación no es estadísticamente distinta del resto de las especificaciones, indican-

15 Meese y Rogoff (1983a,b) encuentran que una gran cantidad de modelos empíricos para el tipo de cambio nominal no son capaces de generar proyecciones fuera de muestra más precisas respecto de aquellas generadas por una caminata aleatoria.

16 Este resultado es consistente con Diebold y Li (2006), en el sentido de que la caminata aleatoria tiende a generar "mejores" proyecciones que modelos empíricos alternativos sólo dentro de horizontes de proyección reducidos. 


\section{TABLA 7}

TEST DIEBOLD Y MARIANO SOBRE PROYECCIONES ICV DE CONSUMO

\begin{tabular}{|c|c|c|c|c|}
\hline & \multicolumn{4}{|c|}{ Horizonte de Proyección } \\
\hline & 3 meses & 6 meses & 12 meses & 24 meses \\
\hline$E\left[d_{1,2}\right]$ & $1,581 * * *$ & $0,906 * * *$ & $0,882 * *$ & $1,315 * *$ \\
\hline$E\left[d_{1,3}\right]$ & $1,634 * * *$ & $0,987 * * *$ & $1,117^{* *}$ & $2,158 * *$ \\
\hline$E\left[d_{1,4}\right]$ & 0,137 & $-0,953$ & 0,118 & 0,368 \\
\hline$E\left[d_{1,5}\right]$ & $1,461 * * *$ & 0,323 & 0,753 & $1,419 *$ \\
\hline$E\left[d_{1,6}\right]$ & $1,753 * * *$ & 0,627 & 0,858 & $1,970 * *$ \\
\hline$E\left[d_{1,7}\right]$ & $1,881 * * *$ & $1,010^{* *}$ & 0,279 & $-3,671 * *$ \\
\hline$E\left[d_{2,3}\right]$ & 0,052 & $0,081 *$ & $0,235^{*}$ & $0,844 *$ \\
\hline$E\left[d_{2,4}\right]$ & $-1,444 * * *$ & $-1,859 * * *$ & $-0,764$ & $-0,947 * * *$ \\
\hline$E\left[d_{2,5}\right]$ & $-0,121$ & $-0,583 * *$ & $-0,129$ & 0,104 \\
\hline$E\left[d_{2,6}\right]$ & $0,172 * *$ & $-0,279$ & $-0,024$ & 0,656 \\
\hline$E\left[d_{27}\right]$ & $0,299 * * *$ & 0,103 & $-0,603$ & $-4,986 * *$ \\
\hline$E\left[d_{3,4}\right]$ & $-1,496 * * *$ & $-1,940 * * *$ & $-0,998 *$ & $-1,790 * * *$ \\
\hline$E\left[d_{3,5}\right]$ & $-0,173 * * *$ & $-0,664 * * *$ & $-0,364 *$ & $-0,740 * * *$ \\
\hline$E\left[d_{3,6}\right]$ & $0,120 * * *$ & $-0,360^{*}$ & $-0,259 * *$ & $-0,188 * *$ \\
\hline$E\left[d_{3,7}\right]$ & $0,247 * *$ & 0,023 & $-0,838$ & $-5,829 * *$ \\
\hline$E\left[d_{4,5}\right]$ & $1,323 * * *$ & 1,276 *** & 0,653 & 1,050 *** \\
\hline$E\left[d_{4,6}\right]$ & $1,616^{* * *}$ & $1,580 * * *$ & 0,740 & $1,602 * * *$ \\
\hline$E\left[d_{4,7}\right]$ & $1,743 * * *$ & $1,963 * * *$ & 0,160 & $-4,039 *$ \\
\hline$E\left[d_{5,6}\right]$ & $0,293 * * *$ & $0,304 * * *$ & 0,105 & $0,552 * * *$ \\
\hline$E\left[d_{5,7}\right]$ & $0,420 * * *$ & $0,687 * * *$ & $-0,474$ & $-5,089 * *$ \\
\hline$E\left[d_{6,7}\right]$ & $0,127^{*}$ & $0,383 * *$ & $-0,579$ & $-5,641 * *$ \\
\hline
\end{tabular}

Nota: $\quad *, * *, * * *$ implican diferenciales de pérdida significativos al $10 \%, 5 \%$ y $1 \%$, respectivamente.

do que todos los modelos evaluados generan proyecciones igualmente precisas, al menos en el corto plazo. Si se considera un horizonte de proyección a 24 meses, las especificaciones (3) y (6) son las que poseen un menor error de proyección y, al igual que para la cartera de consumo, dichas modelaciones son las que poseen los menores criterios de información $A I C$ y $B I C$.

Para el caso de la cartera hipotecaria (Tabla 9), los resultados muestran que para cualquier horizonte de proyección la especificación (3) es la que genera las proyecciones más precisas, aunque dicha ventaja se torna menos clara dentro de horizontes menores a 6 meses, en el sentido que las modelaciones (2) y (4) -e interesantemente la caminata aleatoria no- aparecen como alternativas igualmente válidas desde un punto de vista del diferencial de pérdida.

En resumen, la aplicación del test Diebold y Mariano sobre las proyecciones fuera de muestra de cada especificación y cada cartera de colocaciones revela dos aspectos relacionados con la identificación de las modelaciones de mayor poder predictivo: (i) el test no es totalmente capaz de identificar una única especificación favorita (o al menos un par) dentro de horizontes de proyección menores a 12 meses; y (ii) para un horizonte de 2 años, las especificaciones que generan proyecciones fuera de muestra más precisas son justamente aquellas que mejor ajustan los datos en la muestra. En 
TABLA 8

TEST DIEBOLD Y MARIANO SOBRE PROYECCIONES ICV COMERCIAL

\begin{tabular}{|llccc|}
\hline & \multicolumn{4}{c}{ Horizonte de Proyección } \\
\cline { 2 - 4 } & 3 meses & 6 meses & 12 meses & 24 meses \\
\hline$E\left[d_{1,2}\right]$ & $-0,005$ & 0,005 & 0,011 & $0,061^{*}$ \\
$E\left[d_{1,3}\right]$ & $-0,023^{* * *}$ & $-0,008$ & 0,014 & $0,084^{* *}$ \\
$E\left[d_{1,4}\right]$ & $-0,201^{* * *}$ & $-0,193^{* * *}$ & $-0,079$ & 0,018 \\
$E\left[d_{1,5}\right]$ & $-0,059$ & $-0,075^{* *}$ & 0,018 & 0,077 \\
$E\left[d_{1,6}\right]$ & $-0,077^{* *}$ & $-0,099^{* * *}$ & 0,034 & $0,090^{*}$ \\
$E\left[d_{1,7}\right]$ & $-0,003$ & 0,004 & 0,047 & 0,012 \\
$E\left[d_{2,3}\right]$ & $-0,018^{* * *}$ & $-0,013^{* * *}$ & 0,003 & $0,023 * * *$ \\
$E\left[d_{2,4}\right]$ & $-0,196^{* * *}$ & $-0,198^{* * *}$ & $-0,091$ & $-0,043$ \\
$E\left[d_{2,5}\right]$ & $-0,053$ & $-0,080^{* * *}$ & 0,007 & 0,016 \\
$E\left[d_{2,6}\right]$ & $-0,072^{* * *}$ & $-0,104^{* * *}$ & 0,022 & 0,029 \\
$E\left[d_{2,7}\right]$ & 0,003 & $-0,001$ & 0,035 & $-0,049$ \\
$E\left[d_{3,4}\right]$ & $-0,178^{* * *}$ & $-0,185^{* * *}$ & $-0,094$ & $-0,066$ \\
$E\left[d_{3,5}\right]$ & $-0,035$ & $-0,067^{* *}$ & 0,004 & $-0,007$ \\
$E\left[d_{3,6}\right]$ & $-0,054^{* * *}$ & $-0,091^{* * *}$ & 0,019 & 0,006 \\
$E\left[d_{3,7}\right]$ & $0,021^{* *}$ & $0,012^{*}$ & 0,032 & $-0,072$ \\
$E\left[d_{4,5}\right]$ & $0,143^{* * *}$ & $0,118^{* * *}$ & 0,098 & 0,059 \\
$E\left[d_{4,6}\right]$ & 0,124 & $0,094^{* * *}$ & 0,113 & 0,072 \\
$E\left[d_{4,7}\right]$ & $0,198^{* * *}$ & $0,197^{* * *}$ & $0,126^{*}$ & $-0,006$ \\
$E\left[d_{5,6}\right]$ & $-0,019$ & $-0,024$ & 0,015 & 0,014 \\
$E\left[d_{5,7}\right]$ & 0,056 & $0,079^{* * *}$ & 0,028 & $-0,065$ \\
$E\left[d_{6,7}\right]$ & $0,074^{* * *}$ & $0,103^{* * *}$ & 0,013 & $-0,078$ \\
\hline
\end{tabular}

Nota: $* * *, * * *$ implican diferenciales de pérdida significativos al $10 \%, 5 \%$ y $1 \%$, respectivamente.

conclusión, la evaluación de la capacidad predictiva se realizará dentro de un horizonte de 24 meses y en este contexto las especificaciones con menor error de proyección para la cartera de consumo, comercial e hipotecaria son (3), (6) y (3), respectivamente. Las proyecciones obtenidas a partir de estas modelaciones, para el período julio 2008 a junio 2010, se muestran en la Figura 4.

De la Figura 4 se puede apreciar que las especificaciones identificadas como favoritas generan proyecciones puntuales bastante similares al influjo de cartera vencida observado. Para el caso de la cartera de consumo, si bien el modelo empírico tiende inicialmente a subestimar el $I C V$ de esta cartera, a partir de la segunda mitad de 2009 se puede notar -con un 5\% de significancia- que las proyecciones no son estadísticamente distintas respecto del ICV observado. Para el caso de la cartera comercial, el comportamiento es contrario: las proyecciones generadas por el modelo empírico al inicio de la muestra no son estadísticamente distintas del influjo de cartera vencida efectivo, logrando incluso capturar el excesivo aumento de riesgo observado en junio de 2009 (2,5\%). A partir de esta fecha, el modelo tiende a empeorar su poder predictivo, debido a que la mayor volatilidad observada en el $I C V$ comercial no es totalmente capturada por las proyecciones de la especificación favorita. 
TABLA 9

TEST DIEBOLD Y MARIANO SOBRE PROYECCIONES ICV HIPOTECARIO

\begin{tabular}{|lcccc|}
\hline & \multicolumn{4}{c|}{ Horizonte de Proyección } \\
\cline { 2 - 5 } & 3 meses & 6 meses & 12 meses & 24 meses \\
\hline$E\left[d_{1,2}\right]$ & 0,029 & 0,018 & $-0,071^{*}$ & 0,020 \\
$E\left[d_{1,3}\right]$ & $0,061^{*}$ & 0,029 & $-0,022$ & 0,053 \\
$E\left[d_{1,4}\right]$ & $0,036^{* *}$ & 0,001 & $-0,001$ & $-0,070^{*}$ \\
$E\left[d_{1,5}\right]$ & 0,030 & 0,008 & $-0,083^{* *}$ & $-0,023$ \\
$E\left[d_{1,6}\right]$ & 0,031 & 0,002 & $-0,043^{*}$ & 0,008 \\
$E\left[d_{1,7}\right]$ & $-0,141^{* *}$ & $-0,135^{* * *}$ & $-0,155^{* * *}$ & $-0,191^{* * *}$ \\
$E\left[d_{2,3}\right]$ & $0,032^{* *}$ & 0,011 & $0,049^{* *}$ & $0,033^{* * *}$ \\
$E\left[d_{2,4}\right]$ & 0,007 & $-0,017$ & 0,070 & $-0,090$ \\
$E\left[d_{2,5}\right]$ & 0,001 & $-0,010$ & $-0,012$ & $-0,043^{* *}$ \\
$E\left[d_{2,6}\right]$ & 0,001 & $-0,016$ & 0,028 & $-0,012$ \\
$E\left[d_{2,7}\right]$ & $-0,170^{*}$ & $-0,153^{* * *}$ & $-0,084^{*}$ & $-0,211^{* *}$ \\
$E\left[d_{3,4}\right]$ & $-0,025$ & $-0,028$ & 0,021 & $-0,123$ \\
$E\left[d_{3,5}\right]$ & $-0,031^{* * *}$ & $-0,021^{*}$ & $-0,061^{* * *}$ & $-0,076^{* * *}$ \\
$E\left[d_{3,6}\right]$ & $-0,030^{* *}$ & $-0,027^{* * *}$ & $-0,021^{* * *}$ & $-0,046^{* *}$ \\
$E\left[d_{3,7}\right]$ & $-0,202^{* *}$ & $-0,164^{* * *}$ & $-0,133^{* * *}$ & $-0,244^{* * *}$ \\
$E\left[d_{4,5}\right]$ & $-0,006$ & 0,007 & $-0,082^{* *}$ & 0,047 \\
$E\left[d_{4,6}\right]$ & $-0,005$ & 0,000 & $-0,042^{*}$ & 0,078 \\
$E\left[d_{4,7}\right]$ & $-0,177^{* * *}$ & $-0,136^{* * *}$ & $-0,154^{* * *}$ & $-0,121^{* *}$ \\
$E\left[d_{5,6}\right]$ & 0,001 & $-0,006$ & $0,040^{*}$ & $0,031^{* *}$ \\
$E\left[d_{5,7}\right]$ & $-0,171^{* *}$ & $-0,143^{* * *}$ & $-0,072$ & $-0,168^{* *}$ \\
$E\left[d_{6,7}\right]$ & $-0,172^{* *}$ & $-0,136^{* * *}$ & $-0,112^{* *}$ & $-0,197^{* *}$ \\
\hline
\end{tabular}

Nota: $*, * *, * * *$ implican diferenciales de pérdida significativos al $10 \%, 5 \%$ y $1 \%$, respectivamente.

Finalmente, notamos que si bien las proyecciones asociadas a la cartera hipotecaria logran capturar el aumento sostenido del $I C V$ a partir del segundo semestre de 2008 y hasta comienzos de 2009, la especificación favorita genera proyecciones "más suaves" respecto de la serie efectiva, lo que sería consecuencia del bajo ajuste global del modelo empírico estimado.

\section{CONCLUSIONES}

La evidencia empírica señala al riesgo de crédito como uno de los principales riesgos al cual los sistemas bancarios se encuentran expuestos, además de ser una de las principales causas de las crisis bancarias tanto de carácter sistémico como individual. Esta evidencia pone de manifiesto la importancia de contar con medidas de riesgo de crédito adecuadas para que bancos centrales y/o instituciones de supervisión accionen mecanismos que permitan minimizar la probabilidad de ocurrencia de crisis bancarias y sus potenciales costos asociados.

En este espíritu, el presente artículo propone al influjo de cartera vencida (ICV), definido como la variación del stock de cartera vencida ajustada por castigos y norma- 


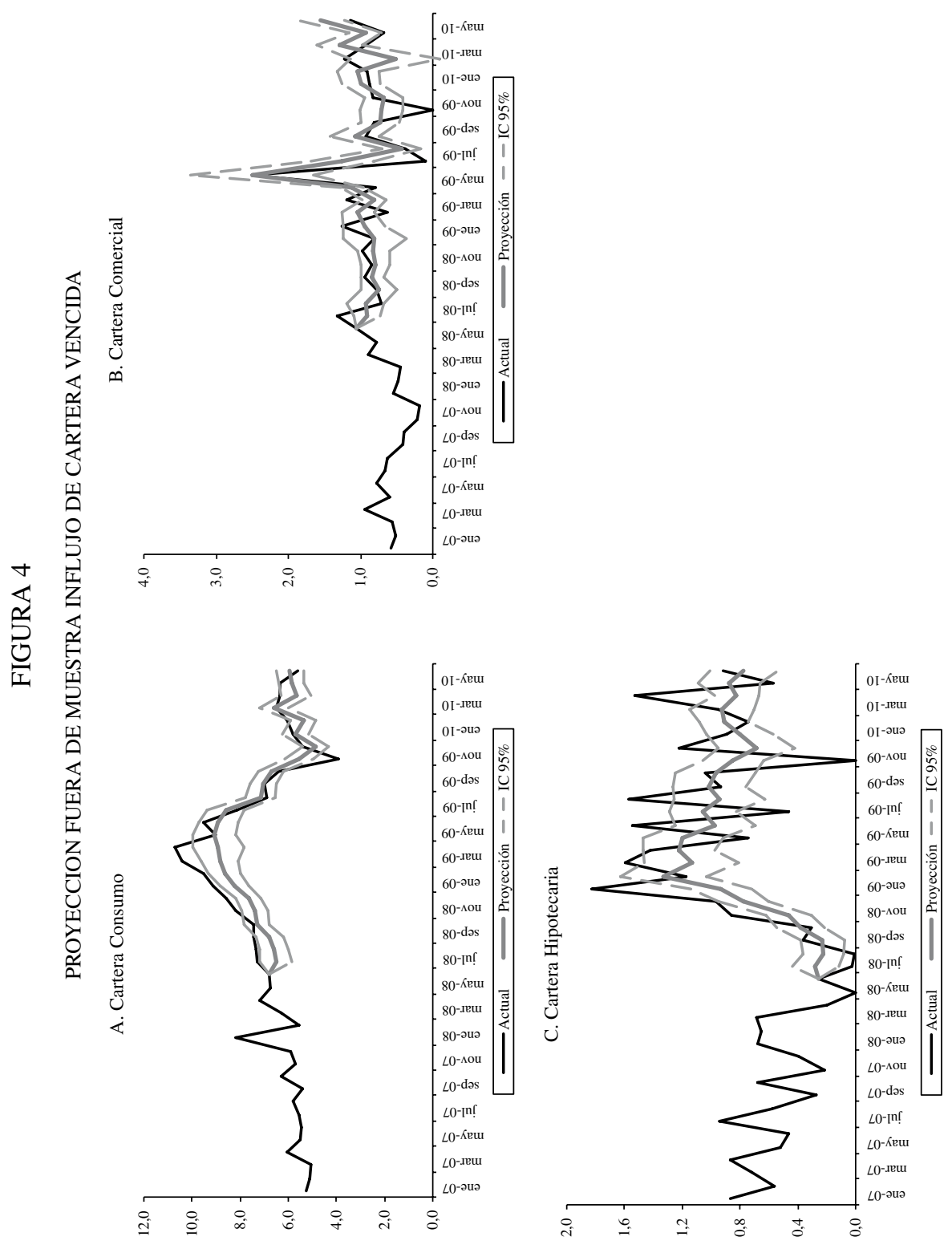


lizada por colocaciones, como principal medida a emplear para modelar el riesgo de crédito de la banca nacional. En particular, el estudio identifica ventajas estadísticas y conceptuales de la medida propuesta, respecto del gasto en provisiones $(G P R)$, que apoyan esta idea. En primer lugar, el $I C V$ detallado por cartera de colocaciones posee una mayor cobertura temporal que el gasto en provisiones ( 7 años, equivalentes a un total de 84 observaciones mensuales adicionales). En segundo lugar, la naturaleza forwardlooking de $G P R$-una de sus principales ventajas sobre $I C V$ - es aplicable sólo a partir del año 2004 debido a los cambios en la normativa de provisiones chilena. Tercero, el gasto en provisiones es de carácter discrecional, conclusión que se desprende del hecho de que la constitución de provisiones es realizada por los bancos evaluadores en base a su aversión relativa al riesgo. Cuarto, el $I C V$ genera series más suaves, respecto de $G P R$, para las carteras de consumo e hipotecaria. Por último, la estructura dinámica de ambas series no presenta diferencias estadísticamente significativas.

El modelo econométrico estimado para el período comprendido entre enero de 1997 a junio de 2010 revela que el influjo de cartera vencida posee relaciones estadísticamente significativas con agregados macroeconómicos como la variación anual del IMACEC, la tasa de interés de corto y largo plazo, la tasa de inflación anual, el tipo de cambio peso-dólar, y las sorpresas en el crecimiento de las colocaciones.

Finalmente, las proyecciones fuera de muestra obtenidas a partir de las especificaciones señalan diferencias económicamente significativas con el $I C V$ efectivo sólo para el caso de las colocaciones hipotecarias, mientras que para las carteras restantes la evolución del influjo de cartera vencida observada durante el período julio 2008 a junio 2010 no difiere significativamente de aquella predicha por el modelo econométrico estimado.

\section{REFERENCIAS}

ADASME, O., G. MAJNONI y M. URIBE (2006). "Calibración del Riesgo de Crédito en los Países Emergentes: La Experiencia de Chile", Serie Técnica de Estudios, Superintendencia de Bancos e Instituciones Financieras.

ALFARO, R., D. CALVO y D. ODA (2009). "Riesgo de Crédito de la Banca de Consumo", Revista Economía Chilena 12(3), pp. 59-77.

ALFARO, R., N. GALLARDO y R. STEIN (2010). "The Determinants of Household Debt Default", Documento de Trabajo Nr. 574, Banco Central de Chile.

ALFARO, R. y A. SAGNER (2011). "Stress Tests for Banking Sector: A Technical Note", Money Affairs 24(2), pp. 143-162.

BLOEM, A. M. y R. FREEMAN (2005). “The Treatment of Nonperforming Loans”, Issue Paper Prepared for the July 2005 Meeting of the Advisory Expert Group on National Accounts, Fondo Monetario Internacional.

BORIO, C. y M. DREHMANN (2009). “Assessing the Risk of Banking Crises”, BIS Quarterly Review, Bank for International Settlements, pp. 29-46.

BÜHLMANN, P. (1997). "Sieve Bootstrap for Time Series", Bernoulli 3(2), pp. 123-148.

CORTAVARRIA, L., C. DZIOBEK, A. KANAYA e I. SONG (2000). "Loan Review, Provisioning and Macroeconomic Linkages", Documento de Trabajo Nr. 195, Fondo Monetario Internacional.

DELL'ARICCIA, G., E. DETRAGIACHE y R. RAJAN (2008). "The Real Effect of Banking Crises", Journal of Financial Intermediation 17(1), pp. 89-112. 
DEMIRGÜC-KUNT, A. y E. DETRAGIACHE (1998). "The Determinants of Banking Crises in Developed and Developing Countries", IMF Staff Paper 45(1), pp. 81-109.

DIEBOLD, F. X. y C. LI (2006). "Forecasting the Term Structure of Government Bond Yields", Journal of Econometrics 130, pp. 337-364.

DIEBOLD, F. X. y R. S. MARIANO (1995). "Comparing Predictive Accuracy”, Journal of Business and Economic Statistic 13, pp. 253-265.

DREHMANN, M. (2009). "Macroeconomic Stress-Testing Banks: A Survey of Methodologies", en StressTesting The Banking System: Methodologies and Applications, pp. 37-67, Cambridge University Press.

ERGUNGOR O. E. y J. B. THOMSON (2005). "Systemic Banking Crises”, Policy Discussion Paper Nr. 90, Banco de la Reserva Federal de Cleveland.

GOLDSTEIN, M. y P. TURNER (1996). "Banking Crises in Emerging Countries: Origins and Policy Options", Documento de Trabajo Nr. 46, Bank for International Settlements.

HARDY, D. C. y C. PAZARBASIOGLU (1999). "Determinants and Leading Indicators of Banking Crises: Further Evidence", IMF Staff Paper 46(3), pp. 247-258.

HOGGARTH, G., R. REIS y V. SAPORTA (2001). "Costs of Banking System Instability: Some Empirical Evidence", Documento de Trabajo Nr. 144, Banco de Inglaterra.

JARA, A. (2005). "Provisiones Bancarias y Ciclo Económico: El Caso de Chile", Informe de Estabilidad Financiera, Primer Semestre, Banco Central de Chile.

JARA, A., L. LUNA y D. ODA (2007). "Pruebas de Tensión de la Banca en Chile", Informe de Estabilidad Financiera, Segundo Semestre, Banco Central de Chile.

MATUS, J. M. (2007). "Indicadores de Riesgo de Crédito: Evolución de la Normativa", Mimeo, Banco Central de Chile.

MATUS, J. M., N. SILVA, A. MARINOVIC y K. FLORES (2010). "Una Visión Global de la Deuda Financiera de los Hogares Chilenos en la Última Década," Estudios Económicos Estadísticos Nr. 81, Banco Central de Chile.

MEESE, R. A. y K. ROGOFF (1983a). "Empirical Exchange Rate Models of the Seventies: Do They Fit Out of Sample?", Journal of International Economics 14(2), pp. 3-24.

MEESE, R. A. y K. ROGOFF (1983b). "The Out of Sample Failure of Empirical Exchange Rate Models: Sampling Error or Misspecification?", en Exchange Rates and International Macroeconomics, pp. 67105, University of Chicago Press.

MOODY'S (2003). "Non-Performing Loans and Loan-Loss Provisioning Policies in Various European Countries", Comentario Especial, Octubre.

RINALDI, L. y A. SANCHIS-ARELLANO (2006). "Household Debt Sustainability: What Explains Household Non-Performing Loans? An Empirical Analysis", Documento de Trabajo Nr. 570, Banco Central Europeo.

SCHULARICK, M. y A. M. TAYLOR (2009). "Credit Booms Gone Bust: Monetary Policy, Leverage Cycles and Financial Crises, 1870-2008”, Documento de Trabajo Nr. 15512, National Bureau of Economic Research.

SHU, C. (2002). "The Impact of Macroeconomic Environment on the Asset Quality of Hong Kong's Banking Sector", Mimeo, Hong Kong Monetary Authority. 


\section{A. ANEXO}

\section{A.1. DETALLE DE VARIABLES EMPLEADAS}

\begin{tabular}{|c|c|c|}
\hline Variable & Fuente & Descripción \\
\hline Cartera Vencida & SBIF & Stock de cartera vencida \\
\hline Castigos & SBIF & Flujo de créditos castigados \\
\hline Colocaciones & SBIF & Colocaciones de créditos \\
\hline IMACEC & $\mathrm{BCCh}$ & Serie empalmada. Variación anual \\
\hline Tasa de Desempleo & INE & Serie empalmada y desestacionalizada \\
\hline Inflación & INE & Variación anual del IPC \\
\hline Tasa de Interés & $\mathrm{BCCh}$ & $\begin{array}{l}\text { Tasa de interés de captación de } 1 \text { a } 3 \text { años } \\
\text { nominal }\end{array}$ \\
\hline $\begin{array}{l}\text { Tasa Mutuos Hipotecarios } \\
\text { Endosables }\end{array}$ & SBIF & $\begin{array}{l}\text { Serie empalmada con tasa de interés de } \\
\text { letras de crédito }\end{array}$ \\
\hline IPSA & Bloomberg & Variación mensual \\
\hline Tipo de Cambio Nominal & $\mathrm{BCCh}$ & $\begin{array}{l}\text { Variación mensual del tipo de cambio } \\
\text { peso-dólar }\end{array}$ \\
\hline $\begin{array}{l}\text { Sorpresas Crecimiento } \\
\text { Colocaciones }\end{array}$ & Elaboración propia & $\begin{array}{l}\text { Diferencia entre variación mensual de } \\
\text { colocaciones efectivas y proyectadas por } \\
\text { proceso } \mathrm{AR}(1)\end{array}$ \\
\hline
\end{tabular}

